\title{
ON THE "TERRA INCOGNITA" FOR THE NEWTON-KANTROVICH METHOD WITH APPLICATIONS
}

\author{
Ioannis Konstantinos Argyros, Yeol Je Cho, and Santhosh George
}

\begin{abstract}
In this paper, we use Newton's method to approximate a locally unique solution of an equation in Banach spaces and introduce recurrent functions to provide a weaker semilocal convergence analysis for Newton's method than before [1]-[13], in some interesting cases, provided that the Fréchet-derivative of the operator involved is $p$-Hölder continuous $(p \in(0,1])$. Numerical examples involving two boundary value problems are also provided.
\end{abstract}

\section{Introduction}

In this study, we are concerned with the problem of approximating a locally unique solution $x^{\star}$ of the nonlinear equation

$$
F(x)=0,
$$

where $F$ is a Fréchet-differentiable operator such that $F^{\prime}$ is a $p$-Hölder continuous operator $(p \in(0,1])$ defined on an open subset $\mathcal{D}$ of a Banach space $\mathcal{X}$ with values in a Banach space $\mathcal{Y}$. A large number of problems in applied mathematics and also in engineering are solved by finding the solutions of certain equations. For example, dynamic systems are mathematically modeled by difference or differential equations and their solutions usually represent the states of the systems. For the sake of simplicity, assume that a time-invariant system is driven by the equation $\dot{x}=Q(x)$ (for some suitable operator $Q$ ), where $x$ is the state. Then the equilibrium states are determined by solving the equation (1.1). Similar equations are used in the case of discrete systems. The unknowns of engineering equations can be functions (difference, differential, and integral equations), vectors (systems of linear or nonlinear algebraic equations) or real or complex numbers (single algebraic equations with single unknowns). Except in special cases, the most commonly used solution methods are iterative when starting from one or several initial approximations a sequence is constructed

Received February 20, 2013; Revised August 14, 2013.

2010 Mathematics Subject Classification. 65H10, 65G99, 65J15, 47H17, 49M15.

Key words and phrases. Newton's method, Banach space, recurrent functions, Hölder continuity, Lipschitz continuity, semilocal convergence, Newton-Kantorovich hypothesis, differential equation. 
that converges to a solution of the equation. Iteration methods are also applied for solving optimization problems. In such cases, the iteration sequences converge to an optimal solution of the problem at hand. Since all of these methods have the same recursive structure, they can be introduced and discussed in a general framework. We study the convergence of Newton's method

$$
x_{n+1}=x_{n}-F^{\prime}\left(x_{n}\right)^{-1} F\left(x_{n}\right) \quad\left(n \geq 0, x_{0} \in \mathcal{D}\right) .
$$

A survey of sufficient conditions for the local as well as the semilocal convergence of Newton-type methods as well as an error analysis for such methods can be found in [1]-[5], [9] and the references there.

In this paper, we introduce our new idea of recurrent functions to provide a finer semilocal convergence for Newton's method (1.2) than before [1]-[13]. Also, numerical examples involving differential equations are also provided in this study.

\section{Preliminaries and background}

To make the study as self-contained as possible, we briefly reintroduce some results.

Let $x_{0} \in \mathcal{D}$ be such that $F^{\prime}\left(x_{0}\right)^{-1} \in \mathcal{L}(\mathcal{Y}, \mathcal{X})$ (the space of bounded linear operators from $\mathcal{Y}$ into $\mathcal{X})$. Assume that $F^{\prime}$ satisfies the center-Hölder condition

$$
\left\|F^{\prime}\left(x_{0}\right)^{-1}\left(F^{\prime}(x)-F^{\prime}\left(x_{0}\right)\right)\right\| \leq \ell_{0}\left\|x-x_{0}\right\|^{p} \quad\left(\ell_{0}>0\right)
$$

and the Hölder condition

$$
\left\|F^{\prime}\left(x_{0}\right)^{-1}\left(F^{\prime}(x)-F^{\prime}(y)\right)\right\| \leq \ell\|x-y\|^{p} \quad(\ell>0)
$$

for all $x, y \in U\left(x_{0}, R\right)=\left\{x \in \mathcal{X}:\left\|x-x_{0}\right\|<R, R>0\right\} \subseteq \mathcal{D}$.

Note that, in general,

$$
\ell_{0} \leq \ell
$$

holds and $\frac{\ell}{\ell_{0}}$ can be arbitrarily large [4] (see also Examples 5.2-5.6). Define the following:

and a function

$$
\eta \geq\left\|F^{\prime}\left(x_{0}\right)^{-1} F\left(x_{0}\right)\right\|, \quad h=\ell \eta^{p}
$$

$$
\psi(r)=\frac{\ell}{1+p} r^{1+p}-r+\eta
$$

The first semilocal convergence result for Newton's and modified Newton's methods under Hölder conditions were given in [12] and [13]:

Theorem 2.1. Assume that

$$
h \leq\left(\frac{p}{1+p}\right)^{p}, \quad r^{*} \leq R,
$$

where $r^{*}$ is the smallest positive zero of function $\psi$. Then sequence $\left\{x_{n}\right\}$ generated by modified Newton's method:

$$
y_{n+1}=y_{n}-F^{\prime}\left(y_{0}\right)^{-1} F\left(y_{n}\right) \quad\left(y_{0}=x_{0}, n \geq 0\right)
$$


is well defined, remains in $U\left(x_{0}, r^{*}\right)$ for all $n \geq 0$ and converges to a unique solution $x^{\star}$ of the equation (1.1) in $U\left(x_{0}, r^{*}\right)$.

Moreover, if

$$
h \leq h_{\nu},
$$

where $h_{\nu}$ is the unique solution in $(0,1)$ of the equation $\left(\frac{t}{1+p}\right)^{p}=(1-t)^{1+p}$, then Newton's method (1.2) converges to $x^{\star}$ as well.

In the Lipschitz case $p=1$, the conditions (2.4) and (2.5) reduce to the famous Newton-Kantorovich hypothesis [10]:

$$
h_{K}=\ell \eta \leq \frac{1}{2}
$$

Later, in [4], [7], [9] and [11], a new approach was used based on the idea is to optimize $b$ in the equation $\psi_{b}(r)=\frac{b \ell}{1+p} r^{1+p}-r+\eta=0$ for some $b \geq 1$ assuming that $h \leq \frac{1}{b}\left(\frac{p}{1+p}\right)^{p}$.

The preceding condition guarantees the equation is solvable (see Proposition 1.1 in [8] or [9]). In [4, p. 190], we showed that the parameter $b$ can be replaced by the smaller:

$$
c(p, d)=d+\left(\frac{p}{1+p}\right)^{p} \quad\left(d=\frac{\ell_{0}}{\ell}\right) .
$$

Moreover, we showed that our majorizing sequence is finer and the information on the location of the solution is at least as precise as in [8] and [9].

Recently, a new result was given in [7], which improves earlier sufficient convergence conditions for all $p \in(0,1)$, but not necessarily the error bounds [8]-[9] and [11]-[13]. Let

$$
c_{0}=\frac{\ell+\sqrt{\ell^{2}+4 \ell_{0} \ell(1+p)^{p} p^{1-p}}}{2 \ell}
$$

and

$$
h_{0}(t)=\left(1-\frac{1}{t}\right)^{p} \frac{1+p}{\left(\left(\ell_{0}(1+p)\right)^{\frac{1}{1-p}}+(\ell t(t-1))^{\frac{1}{1-p}}\right)^{1-p}} .
$$

Then the condition is for $b \geq\left\|F^{\prime}\left(x_{0}\right)\right\|^{-1}$ :

$$
\eta^{p} b \leq h_{0}\left(c_{0}\right) .
$$

We provide new sufficient convergence conditions with error bounds that can be better than the ones in [7] (see also Examples 5.2-5.6). In order for us to achieve this task, we note that the results in [7] can be given in affine invariant form by simply replacing $F$ by $F^{\prime}\left(x_{0}\right)^{-1} F$. Therefore, the condition (2.8) becomes

$$
\eta \leq\left(h_{0}\left(c_{0}\right)\right)^{\frac{1}{p}} .
$$

In the Lipschitz case $(p=1)$ (not covered in [7]), we show that the famous Newton-Kantorovich hypothesis (2.6) can be weakened. 


\section{Semilocal convergence analysis for Newton's method}

We need the following result on majorizing sequences for Newton's method (1.2).

Lemma 3.1. Let $\ell_{0}>0, \ell>0, p \in(0,1]$ and $\eta>0$ be given constants. Define the constants $\delta, \eta_{1}, \eta \delta_{2}, s_{0}$ and $\eta_{0}$ by:

$$
\begin{gathered}
\delta=\left(\frac{2 \ell}{\ell+\sqrt{\ell^{2}+4 \ell_{0} \ell(1+p)}}\right)^{\frac{1}{p}}(1+p), \quad \eta_{1}=\left(\frac{1+p}{\ell+(1+p) \ell_{0} s_{0}}\right)^{\frac{1}{p}} \frac{\delta}{1+p}, \\
\eta_{2}=\left(\frac{1+p}{\ell+\ell_{0}\left(1+p+s_{0}\right)}\right)^{\frac{1}{p}}, \quad \eta_{0}=\min \left\{\eta_{1}, \eta_{2}\right\},
\end{gathered}
$$

where

$$
s_{0}=\left(\frac{\delta}{1+p}\right)^{p}
$$

Assume that

$$
\eta \leq \eta_{0}
$$

Then the scalar sequence $\left\{t_{n}\right\}$ given by

$$
t_{0}=0, \quad t_{1}=\eta, \quad t_{n+2}=t_{n+1}+\frac{\ell\left(t_{n+1}-t_{n}\right)^{1+p}}{(1+p)\left(1-\ell_{0} t_{n+1}^{p}\right)} \quad(n \geq 0)
$$

is non-decreasing, bounded from above by

$$
t^{* *}=\frac{\eta}{1-s_{0}}
$$

and converges to its unique least upper bound $t^{*}$ such that

$$
\eta \leq t^{*} \leq t^{* *} .
$$

Moreover, the following estimates hold: for all $n \geq 0$,

$$
0 \leq t_{n+2}-t_{n+1} \leq s_{0}\left(t_{n+1}-t_{n}\right) \leq s_{0}^{n+1} \eta .
$$

Proof. The estimate (3.6) shall hold if, by (3.3),

$$
0 \leq t_{k+2}-t_{k+1}=\frac{\ell\left(t_{k+1}-t_{k}\right)^{p} s_{0}}{(1+p)\left(1-\ell_{0} t_{k+1}^{p}\right)}, \quad \ell_{0} t_{k+1}^{p}<1 .
$$

For $k=0$, (3.7) holds by (3.1) and (3.3). Let us assume that (3.6)-(3.7) hold true for all $k \leq n$. Then we obtain

$$
\begin{aligned}
t_{k+1} & \leq t_{k}+s_{0}\left(t_{k}-t_{k-1}\right) \\
& \leq t_{k-1}+s_{0}\left(t_{k-1}-t_{k-2}\right)+s_{0}\left(t_{k}-t_{k-1}\right) \\
& \leq t_{1}+s_{0}\left(t_{1}-t_{0}\right)+s_{0}\left(t_{2}-t_{1}\right)+\cdots+s_{0}\left(t_{k}-t_{k-1}\right) \\
& \leq \eta+s_{0} \eta+s^{2} \eta+\cdots+s_{0}^{k} \eta \\
& =\frac{1-s_{0}^{k+1}}{1-s_{0}} \eta<\frac{\eta}{1-s_{0}}=t^{\star \star}
\end{aligned}
$$


ON THE "TERRA INCOGNITA" FOR THE NEWTON-KANTROVICH METHOD 255

In view of (3.4) and (3.6), the estimates (3.7) hold if

$$
\ell s_{0}^{k} \eta^{p}+(1+p) s_{0} \ell_{0}\left(\frac{1-s_{0}^{k+1}}{1-s_{0}}\right)^{p} \eta^{p} \leq(1+p) s_{0}
$$

or

$$
\ell s_{0}^{k-1} \eta^{p}+\ell_{0}(1+p)\left(1+s_{0}+\cdots+s_{0}^{k}\right) \eta^{p}-(1+p) \leq 0
$$

since $(1+w)^{p} \leq 1+p w$ for all $w \geq 0$ and $p \in[0,1]$. The estimate (3.10) motivates us to introduce recurrent functions $f_{k}(k \geq 1)$ on $(0, \infty)$ for $s_{0}=s$ :

$$
f_{k}(s)=\left(\ell s^{k-1}+(1+p) \ell_{0}\left(1+s+\cdots+s^{k}\right)\right) \eta^{p}-(1+p) .
$$

We need a relationship between two consecutive $f_{k}$ :

$$
\begin{aligned}
f_{k+1}(s)= & \left(\ell s^{k}+(1+p) \ell_{0}\left(1+s+\cdots+s^{k+1}\right)\right) \eta^{p}-(1+p) \\
= & \ell s^{k-1} \eta^{p}-\ell s^{k-1} \eta^{p} \\
& +(1+p) \ell_{0}\left(1+s+\cdots+s^{k}\right) \eta^{p}-(1+p) \ell_{0}\left(1+s+\cdots+s^{k}\right) \eta^{p} \\
& +\ell s^{k} \eta^{p}+(1+p) \ell_{0}\left(1+s+\cdots+s^{k+1}\right) \eta^{p}-(1+p) \\
= & f_{k}(s)+g(s) \eta^{p},
\end{aligned}
$$

where

$$
g(s)=(1+p) \ell_{0} s^{2}+\ell s-\ell .
$$

Note that $\delta_{0}$ given by (3.2) is the unique positive zero of function $g$.

Now, we show instead of (3.10) that

$$
f_{k}\left(s_{0}\right) \leq 0 \quad(k \geq 1) .
$$

Using (3.11) and (3.12), we get

$$
f_{k}\left(s_{0}\right)=f_{1}\left(s_{0}\right) .
$$

Hence, in view of (3.13) and (3.14), we only need to show that

$$
f_{k}\left(s_{0}\right) \leq 0
$$

which is true by $(3.2)$ and (3.3). Define a function $f_{\infty}$ on $[0, \infty)$ by

$$
f_{\infty}\left(s_{0}\right)=\lim _{k \rightarrow \infty} f_{k}\left(s_{0}\right) \quad(k \geq 1) .
$$

Then we also have

$$
f_{\infty}\left(s_{0}\right)=\lim _{k \rightarrow \infty} f_{k}\left(s_{0}\right) \leq 0 .
$$

This completes the induction for (3.6)-(3.7) and (3.13).

Finally, note that sequence $\left\{t_{n}\right\}$ is non-decreasing and bounded above by $t^{\star \star}$ and so it converges to $t^{\star}$ satisfying (3.5). This completes the proof.

Remark 3.2. We show that the order of convergence for iteration $\left\{t_{n}\right\}$ is quadratic under the conditions of Lemma 3.1 for $p=1$ (see Lemma 5.5). Moreover, for the case $p \in(0,1)$, see Example 5.6. 
From now on, we refer to (2.1), (2.2), (2.3) and hypotheses of Lemma 3.1 as the $(C)$ conditions. We can show the main semilocal convergence theorem for Newton's method (1.2):

Theorem 3.3. Under the $(C)$ conditions, further, suppose that

$$
\bar{U}\left(x_{0}, t^{*}\right) \subseteq U\left(x_{0}, R\right)
$$

holds. Then the sequence $\left\{x_{n}\right\}$ generated by Newton's method (1.2) is well defined, remains in $\bar{U}\left(x_{0}, t^{*}\right)$ for all $n \geq 0$ and converges to a unique solution $x^{\star} \in \bar{U}\left(x_{0}, t^{*}\right)$ of the equation $F(x)=0$. Moreover, the following estimates bounds hold: for all $n \geq 0$,

$$
\left\|x_{n+2}-x_{n+1}\right\| \leq \frac{\ell\left\|x_{n+1}-x_{n}\right\|^{1+p}}{(1+p)\left[1-\ell_{0}\left\|x_{n+1}-x_{0}\right\|^{p}\right]} \leq t_{n+2}-t_{n+1}
$$

and

$$
\left\|x_{n}-x^{\star}\right\| \leq t^{*}-t_{n},
$$

where the iteration $\left\{t_{n}\right\}$ and the point $t^{*}$ are given in Lemma 3.1.

Furthermore, if there exists $R>t^{*}$ such that

$$
R_{0} \leq R, \quad \ell_{0} \int_{0}^{1}\left[\theta t^{*}+(1-\theta) R\right]^{p} d \theta \leq 1,
$$

then the solution $x^{\star}$ is unique in $U\left(x_{0}, R_{0}\right)$.

Proof. We prove that

$$
\left\|x_{k+1}-x_{k}\right\| \leq t_{k+1}-t_{k}, \quad \bar{U}\left(x_{k+1}, t^{*}-t_{k+1}\right) \subseteq \bar{U}\left(x_{k}, t^{*}-t_{k}\right)
$$

hold for all $n \geq 0$. For all $z \in \bar{U}\left(x_{1}, t^{*}-t_{1}\right)$, it follows that

$$
\left\|z-x_{0}\right\| \leq\left\|z-x_{1}\right\|+\left\|x_{1}-x_{0}\right\| \leq t^{*}-t_{1}+t_{1}=t^{*}-t_{0}
$$

implies $z \in \bar{U}\left(x_{0}, t^{*}-t_{0}\right)$. Since also $\left\|x_{1}-x_{0}\right\|=\left\|F^{\prime}\left(x_{0}\right)^{-1} F\left(x_{0}\right)\right\| \leq \eta=t_{1}$, (3.18) hold for $n=0$. Assume that they hold for $n=0,1, \ldots, k$. Then

$$
\left\|x_{k+1}-x_{0}\right\| \leq \sum_{i=1}^{k+1}\left\|x_{i}-x_{i-1}\right\| \leq \sum_{i=1}^{k+1}\left(t_{i}-t_{i-1}\right)=t_{k+1}-t_{0}=t_{k+1}
$$

and

$$
\left\|x_{k}+\theta\left(x_{k+1}-x_{k}\right)-x_{0}\right\| \leq t_{k}+\theta\left(t_{k+1}-t_{k}\right)<t^{*} \quad(\theta \in[0,1]) .
$$

Using (1.2), we obtain the approximation

$$
\begin{aligned}
F\left(x_{k+1}\right) & =F\left(x_{k+1}\right)-F\left(x_{k}\right)-F^{\prime}\left(x_{k}\right)\left(x_{k+1}-x_{k}\right) \\
& =\int_{0}^{1}\left[F^{\prime}\left(x_{k}+\theta\left(x_{k+1}-x_{k}\right)\right)-F^{\prime}\left(x_{k}\right)\right]\left(x_{k+1}-x_{k}\right) d \theta
\end{aligned}
$$

and, by (2.2),

(3.19) $\left\|F^{\prime}\left(x_{0}\right)^{-1} F\left(x_{k+1}\right)\right\|$ 


$$
\begin{aligned}
& \leq \int_{0}^{1}\left\|F^{\prime}\left(x_{0}\right)^{-1}\left[F^{\prime}\left(x_{k}+\theta\left(x_{k+1}-x_{k}\right)\right)-F^{\prime}\left(x_{k}\right)\right]\right\| d \theta\left\|x_{k+1}-x_{k}\right\| \\
& \leq \frac{\ell}{1+p}\left\|x_{k+1}-x_{k}\right\|^{1+p} .
\end{aligned}
$$

By (2.1), the estimate

$$
\left\|F^{\prime}\left(x_{0}\right)^{-1}\left[F^{\prime}\left(x_{k+1}\right)-F^{\prime}\left(x_{0}\right)\right]\right\| \leq \ell_{0}\left\|x_{k+1}-x_{0}\right\|^{p} \leq \ell_{0} t_{k+1}^{p}<1
$$

and the Banach Lemma on invertible operators ([1], [5], [10]), $F^{\prime}\left(x_{k+1}\right)^{-1}$ exists and

$$
\left\|F^{\prime}\left(x_{0}\right) F^{\prime}\left(x_{k+1}\right)^{-1}\right\| \leq \frac{1}{1-\ell_{0}\left\|x_{k+1}-x_{0}\right\|^{p}} \leq \frac{1}{1-\ell_{0} t_{k+1}^{p}} .
$$

Therefore, by (1.2), (3.6), (3.19) and (3.20), we obtain in turn

$$
\begin{aligned}
\left\|x_{k+2}-x_{k+1}\right\| & =\left\|F^{\prime}\left(x_{k+1}\right)^{-1} F\left(x_{k+1}\right)\right\| \\
& \leq\left\|F^{\prime}\left(x_{k+1}\right)^{-1} F^{\prime}\left(x_{0}\right)\right\|\left\|F^{\prime}\left(x_{0}\right)^{-1} F\left(x_{k+1}\right)\right\| \\
& \leq \frac{\ell\left\|x_{k+1}-x_{k}\right\|^{1+p}}{(1+p)\left[1-\ell_{0}\left\|x_{k+1}-x_{0}\right\|^{p}\right]} \\
& \leq \frac{\ell\left(t_{k+1}-t_{k}\right)^{1+p}}{(1+p)\left[1-\ell_{0} t_{k+1}^{p}\right]} \\
& =t_{k+2}-t_{k+1} .
\end{aligned}
$$

Thus, for all $z \in \bar{U}\left(x_{k+2}, t^{*}-t_{k+2}\right)$, we have

$$
\begin{aligned}
\left\|z-x_{k+1}\right\| & \leq\left\|z-x_{k+2}\right\|+\left\|x_{k+2}-x_{k+2}\right\| \\
& \leq t^{*}-t_{k+2}+t_{k+2}-t_{k+2} \\
& =t^{*}-t_{k+1},
\end{aligned}
$$

that is, $z \in \bar{U}\left(x_{k+1}, t^{*}-t_{k+1}\right)$. The estimates (3.21) imply that (3.18) holds for $n=k+1$. By induction, the proof of (3.18) is completed. Lemma 3.1 implies that $\left\{t_{n}\right\}$ is a Cauchy sequence. From (3.18) $\left\{x_{n}\right\}(n \geq 0)$ becomes a Cauchy sequence too and so it converges to some $x^{\star} \in \bar{U}\left(x_{0}, t^{*}\right)$ (since $\bar{U}\left(x_{0}, t^{*}\right)$ is a closed set), so that (3.16) holds. Thus (3.21) yields $F\left(x^{\star}\right)=0$.

Finally, to show the uniqueness, let $y^{\star}$ be a solution of the equation $F(x)=0$ in $U\left(x_{0}, R\right)$. It follows from (2.1), (3.17), as in (3.20),

$$
\begin{aligned}
& \left\|F^{\prime}\left(x_{0}\right)^{-1} \int_{0}^{1}\left[F^{\prime}\left(y^{*}+\theta\left(x^{*}-y^{*}\right)\right)-F^{\prime}\left(x_{0}\right)\right]\right\| d \theta \\
\leq & \ell_{0} \int_{0}^{1}\left\|y^{*}+\theta\left(x^{*}-y^{*}\right)-x_{0}\right\|^{p} d \theta \\
\leq & \ell_{0} \int_{0}^{1}\left[\theta\left\|x^{*}-x_{0}\right\|+(1-\theta)\left\|y^{*}-x_{0}\right\|\right]^{p} d \theta
\end{aligned}
$$




$$
\begin{aligned}
& <\ell_{0} \int_{0}^{1}\left[\theta t^{*}+(1-\theta) R_{0}\right]^{p} d \theta \\
& \leq 1
\end{aligned}
$$

and the Banach Lemma on invertible operators that linear operator $L=$ $\int_{0}^{1} F^{\prime}\left(y^{*}+\theta\left(x^{*}-y^{*}\right)\right) d \theta$ is invertible. Using the identity $0=F\left(y^{*}\right)-F\left(x^{*}\right)=$ $L\left(x^{*}-y^{*}\right)$, we deduce $x^{\star}=y^{\star}$. To show the uniqueness in $\bar{U}\left(x_{0}, t^{*}\right)$, as in (3.22), we get, by Lemma 3.1 ,

$$
\left\|F^{\prime}\left(x_{0}\right)^{-1}\left(L-F^{\prime}\left(x_{0}\right)\right)\right\| \leq \frac{\ell_{0}}{1+p}\left(t^{*}\right)^{1+p}<1,
$$

which implies again $x^{*}=y^{*}$. That completes the proof.

Remark 3.4. In the result that follows, we show that our error bounds on the distances involved are finer and the location of the solution $x^{*}$ at least as precise than earlier results using (3.6) for $\ell=\ell_{0}$. Denote by $\left\{r_{n}\right\}$ such a sequence. The sequence $\left\{r_{n}\right\}$ was used as a majorizing sequence for $\left\{x_{n}\right\}$ in earlier studies such as [1]-[13].

Proposition 3.5 ([4]). Under hypotheses of Theorems 3.3 and (2.7) with $\ell_{0}<$ $\ell$, the following error bounds hold:

$$
\begin{array}{cl}
r_{0}=t_{0}=0, \quad r_{1}=t_{1}=\eta, & t_{n+1}<r_{n+1} \quad(n \geq 1), \\
t_{n+1}-t_{n}<r_{n+1}-r_{n} \quad(n \geq 1), & t^{\star}-t_{n} \leq r^{*}-r_{n} \quad(n \geq 0), \quad t^{*} \leq r^{*} .
\end{array}
$$

Remark 3.6. It follows from the proof of Theorem 3.3 that sequence $\left\{s_{n}\right\}$ given by

$$
s_{0}=0, s_{1}=\eta, s_{n+2}=s_{n+1}+\frac{l_{1}\left(s_{n+1}-s_{n}\right)^{1+p}}{(1+p)\left(1-\ell_{0} s_{n+1}^{p}\right)}(n \geq 0) \text {, }
$$

where

$$
\ell_{1}= \begin{cases}\ell_{0} & \text { if } n=1 \\ \ell & \text { if } n>1\end{cases}
$$

is also a majorizing sequence for $\left\{x_{n}\right\}$ but finer than $\left\{t_{n}\right\}$ for $\ell_{0}<\ell$.

\section{Semilocal convergence analysis for Newton's method}

Let $\ell_{0}>0, \ell>0, p \in(0,1]$ be fixed and set $\gamma=\left(\frac{\ell}{1+p}\right)^{\frac{1}{p}}$. Define a function $q$ on $[0,1]$ by

$$
q(t)=t^{2+p}-\left(1+\delta_{1}\right) t^{1+p}-t+\delta_{1},
$$

where $\gamma_{1}=\gamma \ell_{0}^{-\frac{1}{p}}$. Then we have $q(0)=\delta_{1}>0$ and $q(1)=-1<0$. Thus it follows from the intermediate value theorem that there exists a zero of the function $q$ in $(0,1)$. Denote by $\eta_{q}$ the minimal such zero. Set $s_{0}=\frac{\eta_{q}}{\gamma}$. Then we obtain

$$
\ell_{0}^{\frac{1}{p}}\left(s_{0}+\frac{1}{\gamma} \frac{\left(\gamma s_{0}\right)^{1+p}}{1-\left(\gamma s_{0}\right)^{1+p}}\right)=1
$$


Define the functions $g$ and $f$ on $\left[0, \frac{1}{\delta}\right)$ by

$$
g(s)=\ell_{0}\left(s+\frac{1}{\delta} \frac{(\delta s)^{1+p}}{1-(\delta s)^{1+p}}\right)^{p}-1, \quad f(s)=(1+p) \delta^{p} g(s)+\ell,
$$

respectively. Then we get

$$
f\left(s_{0}\right)=\ell>0 .
$$

Then it follows that the function $f$ has a unique positive zero denoted $\eta_{f} \in$ $\left(0, s_{0}\right]$. Indeed, by the choice of $\gamma$, we have $f(0)=\ell-(1+p) \gamma^{p}<0$, which, together with (4.1) and the intermediate value theorem, implies the existence of $\eta_{f}$. The uniqueness follows from $f^{\prime}(s)>0(s>0)$, that is, $f$ crosses the positive axis only once.

We can show the following lemma on majorizing sequences for Newton's method (1.2).

Lemma 4.1. Assume that

$$
\eta \leq \eta_{f}
$$

Then the scalar sequence $\left\{t_{n}\right\}$ given by (3.3) is increasing, bounded above by

$$
t^{* *}=\eta+\frac{1}{\gamma} \frac{(\gamma \eta)^{1+p}}{\gamma\left(1-(\gamma \eta)^{1+p}\right)}
$$

and converges to its unique least upper bound $t^{\star}$ such that

$$
0 \leq t^{*} \leq t^{* *} \text {. }
$$

Moreover, the following estimates hold: for all $n \geq 0$,

$$
0 \leq t_{n+2}-t_{n+1} \leq \frac{1}{\delta}\left(\delta\left(t_{n+1}-t_{n}\right)\right)^{1+p} \leq \frac{1}{\delta}(\delta \eta)^{(1+p)^{n+1}}
$$

and

$$
0 \leq t^{*}-t_{n+1} \leq \frac{(\delta \eta)^{(1+p)^{n}}}{\delta\left(1-(\delta \eta)^{1+p}\right)}
$$

Proof. We show, using induction on $k$,

$$
0<\frac{\ell\left(t_{k+1}-t_{k}\right)^{1+p}}{(1+p)\left(1-\ell_{0} t_{k+1}^{p}\right)} \leq \delta^{p}\left(t_{k+1}-t_{k}\right)^{1+p} .
$$

The estimate (4.4) holds for $k=0$ by the initial conditions and (4.2). Let us assume that (4.4) holds for all $m \leq k$. We have in turn

$$
\begin{aligned}
t_{k+1} & \leq t_{k}+\frac{1}{\delta}\left(\delta\left(t_{k+1}-t_{k}\right)\right)^{1+p} \\
& \leq t_{1}+\frac{1}{\delta}\left((\delta \eta)^{(1+p)^{1}}+\cdots+(\delta \eta)^{(1+p)^{k}}\right) \\
& \leq \eta+\frac{1}{\delta}\left((\delta \eta)^{1+p}+\cdots+(\delta \eta)^{(1+p)(k)}\right) \\
& =\eta+\frac{1}{\delta}(\delta \eta)^{1+p}\left(1+\cdots+(\delta \eta)^{(1+p)(k-1)}\right)
\end{aligned}
$$




$$
=\eta+\frac{1}{\delta}(\delta \eta)^{1+p} \frac{1-(\delta \eta)^{(1+p) k}}{1-(\delta \eta)^{1+p}} \leq t^{* *} .
$$

In order for us to show (4.4), it suffices to show the following:

$$
\ell \leq(1+p) \delta^{p}\left(1-\ell_{0} t_{k+1}^{p}\right)
$$

or

$$
(1+p) \ell_{0} \delta^{p} t_{k+1}^{p}-(1+p) \delta^{p}+\ell \leq 0
$$

or, by (4.5),

$$
(1+p) \ell_{0} \delta^{p}\left(\eta+\frac{1}{\delta} \frac{(\delta \eta)^{1+p}}{1-(\delta \eta)^{1+p}}\right)^{p}-(1+p) \delta^{p}+\ell \leq 0,
$$

which is true by (4.1) and (4.2). The induction for (4.4) is completed. Hence the sequence $\left\{t_{k}\right\}$ is increasing, bounded above by $t^{* *}$ and so it converges to its unique maximum lowest bound $t^{*} \in\left[0, t^{* *}\right]$.

Finally, to show (4.3), let $m \geq 1$. Then we have in turn

$$
\begin{aligned}
t_{k+m}-t_{k} & \leq\left(t_{k+m}-t_{k+m-1}\right)+\left(t_{k+m-1}-t_{k+m-2}\right)+\cdots+\left(t_{k+1}-t_{k}\right) \\
& \leq \frac{1}{\delta}\left((\delta \eta)^{(1+p)^{k+m}}+(\delta \eta)^{\left.(1+p)^{k+m-1}+\cdots+(\delta \eta)^{(1+p)^{k}}\right)}\right. \\
& =\frac{1}{\delta}\left(1+\cdots+(\delta \eta)^{m(1+p)}\right)(\delta \eta)^{(1+p)^{k}} \\
& =\frac{1}{\delta} \frac{1-(\delta \eta)^{(m+1)(1+p)}}{1-(\delta \eta)^{1+p}}(\delta \eta)^{(1+p)^{k}} .
\end{aligned}
$$

By letting $m \rightarrow \infty$ in (3.6), we obtain (3.3). This completes the proof.

From now on, we refer to (2.1), (2.2) and hypotheses of Lemma 5.1 as the $\left(C^{*}\right)$ conditions. 1.2).

We can show the main semilocal convergence theorem for Newton's method

Theorem 4.2. Under the $\left(C^{*}\right)$ conditions, further, suppose

$$
\bar{U}\left(x_{0}, t^{*}\right) \subseteq U\left(x_{0}, R\right) .
$$

holds. Then the sequence $\left\{x_{n}\right\}$ generated by Newton's method (1.2) is well defined, remains in $\bar{U}\left(x_{0}, t^{*}\right)$ for all $n \geq 0$ and converges to a unique solution $x^{*} \in \bar{U}\left(x_{0}, t^{*}\right)$ of the equation $F(x)=0$. Moreover, the following estimates hold: for all $n \geq 0$,

$$
\left\|x_{n+2}-x_{n+1}\right\| \leq \frac{\ell\left\|x_{n+1}-x_{n}\right\|^{1+p}}{(1+p)\left[1-\ell_{0}\left\|x_{n+1}-x_{0}\right\|^{p}\right]} \leq t_{n+2}-t_{n+1}
$$

and

$$
\left\|x_{n}-x^{*}\right\| \leq t^{*}-t_{n} .
$$


Furthermore, if there exists $R>t^{*}$ such that

$$
R_{0} \leq R, \quad \ell_{0} \int_{0}^{1}\left[\theta t^{\star}+(1-\theta) R\right]^{p} d \theta \leq 1,
$$

then the solution $x^{*}$ is unique in $U\left(x_{0}, R_{0}\right)$. That is, the order of the convergence is $1+p$.

Proof. Simply, replacing Lemma 3.1 by Lemma 4.1, then we get the conclusion.

\section{Special cases and applications}

In this section, we give some examples and applications to illustrate the main results in this paper.

Application 5.1 (Lipschitz case). Let $p=1$. It is simple algebra to show that all hypotheses of Lemma 3.1 hold true provided that

$$
h_{A}=a \eta \leq \frac{1}{2}
$$

where $a=\frac{1}{8}\left(\ell+4 \ell_{0}+\sqrt{\ell^{2}+8 \ell \ell_{0}}\right)$. Then it follows from (2.6) and (5.1) that

$$
h_{K} \leq \frac{1}{2} \quad \Longrightarrow \quad h_{A} \leq \frac{1}{2},
$$

but not necessarily vice verca unless if $\ell_{0}=\ell$.

Example 5.2. Define the scalar function $F$ by

$$
F(x)=c_{0} x+c_{1}+c_{2} \sin e^{c_{3} x}, x_{0}=0,
$$

where $c_{i}, i=1,2,3$, are the given parameters. Then it can easily be seen that, for $c_{3}$ large and $c_{2}$ sufficiently small, $\frac{\ell}{\ell_{0}}$ can be arbitrarily large, that is, (5.1) may be satisfied, but not (2.6).

Example 5.3. Let $\mathcal{X}=\mathcal{Y}=\mathbb{R}^{2}$ equipped with the max-norm, $x_{0}=(1,1)^{T}$, $\left.U_{0}=\left\{x:\left\|x-x_{0}\right\| \leq 1-\beta\right\}, \beta \in\left[0, \frac{1}{2}\right)\right\}$ and define a function $F$ on $U_{0}$ by

$$
F(x)=\left(\xi_{1}^{3}-\beta, \xi_{2}^{3}-\ell\right) x=\left(\xi_{1}, \xi_{1}\right)^{T} .
$$

The Fréchet derivative is given by $F^{\prime}(x)=\left[\begin{array}{cc}3 \xi_{1}^{2} & 0 \\ 0 & 3 \xi_{2}^{2}\end{array}\right]$. Using hypotheses of Theorem 3.3, we get $\eta=\frac{1}{3}(1-\beta), \ell_{0}=3-\beta$ and $\ell=2(2-\beta)$. The Kantorovich condition (2.6) is violated since

$$
\frac{4}{3}(1-\beta)(2-\beta)>1
$$

for all $\beta \in\left[0, \frac{1}{2}\right)$. Hence there is no guarantee that Newton's method (1.2) converges to $x^{\star}=(\sqrt[3]{\beta}, \sqrt[3]{\ell})^{T}$, starting at $x_{0}$. However, our condition (5.1) is true for all $\beta \in I=\left[.450339002, \frac{1}{2}\right)$. Hence the conclusions of our Theorem 3.3 can apply to solve the equation (5.2) for all $\beta \in I$. 
Example 5.4. Consider the following nonlinear boundary value problem ([5]):

$$
\left\{\begin{array}{l}
u^{\prime \prime}=-u^{3}-\gamma u^{2} \\
u(0)=0, \quad u(1)=1
\end{array}\right.
$$

It is well known that this problem can be formulated as the integral equation

$$
u(s)=s+\int_{0}^{1} Q(s, t)\left(u^{3}(t)+\gamma u^{2}(t)\right) d t,
$$

where $Q$ is the Green function:

$$
Q(s, t)= \begin{cases}t(1-s), & t \leq s, \\ s(1-t), & s<t .\end{cases}
$$

We observe that $\max _{0 \leq s \leq 1} \int_{0}^{1}|Q(s, t)|=\frac{1}{8}$. Let $\mathcal{X}=\mathcal{Y}=\mathcal{C}[0,1]$ with the norm $\|x\|=\max _{0 \leq s \leq 1}|x(s)|$. Then the problem (5.3) is in the form (1.1), where a mapping $F: \mathcal{D} \longrightarrow \mathcal{Y}$ is defined by

$$
[F(x)](s)=x(s)-s-\int_{0}^{1} Q(s, t)\left(x^{3}(t)+\gamma x^{2}(t)\right) d t .
$$

It is easy to verify that the Fréchet derivative of $F$ is defined in the form

$$
\left[F^{\prime}(x) v\right](s)=v(s)-\int_{0}^{1} Q(s, t)\left(3 x^{2}(t)+2 \gamma x(t)\right) v(t) d t .
$$

If we set $u_{0}(s)=s$ and $\mathcal{D}=U\left(u_{0}, R\right)$, then, since $\left\|u_{0}\right\|=1$, it is easy to verify that $U\left(u_{0}, R\right) \subset U(0, R+1)$. It follows that $2 \gamma<5$ and so

$$
\begin{aligned}
\left\|I-F^{\prime}\left(u_{0}\right)\right\| & \leq \frac{3\left\|u_{0}\right\|^{2}+2 \gamma\left\|u_{0}\right\|}{8}=\frac{3+2 \gamma}{8}, \\
\left\|F^{\prime}\left(u_{0}\right)^{-1}\right\| & \leq \frac{1}{1-\frac{3+2 \gamma}{8}}=\frac{8}{5-2 \gamma} \\
\left\|F\left(u_{0}\right)\right\| & \leq \frac{\left\|u_{0}\right\|^{3}+\gamma\left\|u_{0}\right\|^{2}}{8}=\frac{1+\gamma}{8} \\
\left\|F\left(u_{0}\right)^{-1} F\left(u_{0}\right)\right\| & \leq \frac{1+\gamma}{5-2 \gamma} .
\end{aligned}
$$

On the other hand, for any $x, y \in \mathcal{D}$, we have

$$
\begin{aligned}
& {\left[\left(F^{\prime}(x)-F^{\prime}(y)\right) v\right](s) } \\
= & -\int_{0}^{1} Q(s, t)\left(3 x^{2}(t)-3 y^{2}(t)+2 \gamma(x(t)-y(t))\right) v(t) d t .
\end{aligned}
$$

Consequently, we have

$$
\begin{aligned}
\left\|F^{\prime}(x)-F^{\prime}(y)\right\| & \leq \frac{\|x-y\|(2 \gamma+3(\|x\|+\|y\|))}{8} \\
& \leq \frac{\|x-y\|\left(2 \gamma+6 R+6\left\|u_{0}\right\|\right)}{8}
\end{aligned}
$$


ON THE "TERRA INCOGNITA" FOR THE NEWTON-KANTROVICH METHOD 263

$$
=\frac{\gamma+6 R+3}{4}\|x-y\|
$$

and

$$
\begin{aligned}
\left\|F^{\prime}(x)-F^{\prime}\left(u_{0}\right)\right\| & \leq \frac{\left\|x-u_{0}\right\|\left(2 \gamma+3\left(\|x\|+\left\|u_{0}\right\|\right)\right)}{8} \\
& \leq \frac{\left\|x-u_{0}\right\|\left(2 \gamma+3 R+6\left\|u_{0}\right\|\right)}{8} \\
& =\frac{2 \gamma+3 R+6}{8}\left\|x-u_{0}\right\| .
\end{aligned}
$$

Therefore, the conditions of Theorem 3.3 hold with

$$
\eta=\frac{1+\gamma}{5-2 \gamma}, \quad \ell=\frac{\gamma+6 R+3}{4(5-2 \gamma)}, \quad \ell_{0}=\frac{2 \gamma+3 R+6}{8(5-2 \gamma)} .
$$

Note also that $\ell_{0}<\ell$.

The proof of the following results can be found in [5] and [6].

Lemma 5.5. Assume that there exist constants $\ell_{0} \geq 0, \ell \geq 0$ with $\ell_{0} \leq \ell$ and $\eta \geq 0$ such that

$$
q_{0}=\bar{\ell} \eta \leq \frac{1}{2}
$$

where

$$
\bar{\ell}=\frac{1}{8}\left(\ell+4 \ell_{0}+\sqrt{\ell^{2}+8 \ell_{0} \ell}\right) .
$$

The inequality in (5.4) is strict if $\ell_{0}=0$. Then the sequence $\left\{t_{k}\right\}$ given by

$$
t_{0}=0, \quad t_{1}=\eta, \quad t_{k+1}=t_{k}+\frac{\ell\left(t_{k}-t_{k-1}\right)^{2}}{2\left(1-\ell_{0} t_{k}\right)} \quad(k \geq 1)
$$

is well defined, nondecreasing, bounded above by $t^{* *}$ and converges to its unique least upper bound $t^{*} \in\left[0, t^{* *}\right]$, where

$$
t^{* *}=\frac{2 \eta}{2-\delta}, \quad 1 \leq \delta=\frac{4 \ell}{\ell+\sqrt{\ell^{2}+8 \ell_{0} \ell}}<2 \quad\left(\ell_{0} \neq 0\right) .
$$

Moreover, the following estimates hold:

$$
\begin{gathered}
\ell_{0} t^{*} \leq 1, \quad 0 \leq t_{k+1}-t_{k} \leq \frac{\delta}{2}\left(t_{k}-t_{k-1}\right) \leq \cdots \leq\left(\frac{\delta}{2}\right)^{k} \eta \quad(k \geq 1), \\
t_{k+1}-t_{k} \leq\left(\frac{\delta}{2}\right)^{k}\left(2 q_{0}\right)^{2^{k}-1} \eta \quad(k \geq 0) \\
0 \leq t^{*}-t_{k} \leq\left(\frac{\delta}{2}\right)^{k} \frac{\left(2 q_{0}\right)^{2^{k}-1} \eta}{1-\left(2 q_{0}\right)^{2^{k}}} \quad\left(2 q_{0}<1, k \geq 0\right)
\end{gathered}
$$


Example 5.6 (Hölder case, $p \neq 1$ ). The corresponding to (3.9) error bounds in [8] and [7] are given by

$$
0 \leq t_{n}-t_{n+1} \leq t_{n}\left(\frac{c_{0}-1}{c_{0}^{n}-1}\right) \quad(n \geq 1) .
$$

Let $\ell=1, \ell_{0}=p=\frac{1}{2}$ and $\eta=.2$. It can easily be seen that (2.9) and the hypotheses of Lemma 3.1 are satisfied. We get

$$
\begin{gathered}
s_{0}=\delta=0.666 \cdots, \eta_{0}=\eta_{1}=0.4444 \cdots, \eta_{2}=0.5184 \cdots, \\
c_{0}=1.326445825, h_{0}\left(c_{0}\right)^{\frac{1}{2}}=0.6666 \cdots
\end{gathered}
$$

and so

$$
\eta<.6666 \text { and } \eta \leq \eta_{0}
$$

That is, (2.9) and (3.3) are satisfied, respectively. Moreover, using (3.4), we get

$$
\begin{aligned}
t_{0} & =0, \quad t_{1}=.2, \quad t_{2}=.276801908, \quad t_{3}=.296056509, \\
t_{4} & =.298503386, \quad t_{5}=.298614405, \quad t_{6}=.298615478
\end{aligned}
$$

Then we get the following comparison table:

TABLE 1. Comparison between bounds (3.4), (3.6) and (5.5)

\begin{tabular}{|c|c|c|c|}
\hline$n$ & $(3.4)$ & $(3.6)$ & $(5.5)$ \\
\hline 2 & .076801908 & .118980595 & .092376043 \\
3 & .019254601 & .072458013 & .0426666 \\
4 & .002447296 & .46497804 & .019706889 \\
5 & .000111019 & .03138214 & .009102222 \\
6 & .000001073 & .021922049 & .004204136 \\
\hline
\end{tabular}

Hence our error bounds (3.4) and (3.6) compare favorably to the ones in [7], i.e., to (5.5).

Note that either (2.9) or (3.3) is not the optimum sufficient convergence conditions for the semilocal convergence of Newton's method in case $p \in(0,1)$. In practice, we shall use the combination of results providing the best estimates.

Finally, note that the earlier conditions (listed above (2.8)) also do not provide a better error bounds since $\ell_{0}<\ell$. 


\section{Conclusion}

We introduced recurrent polynomials and also used a combination of Hölder and center-Hölder conditions, instead of only Hölder or Lipschitz conditions ([1]-[5], [8]-[13]) for studying a semilocal convergence analysis for Newton's method in Banach spaces. This analysis has the following advantages over the works in [1]-[5] and [8]-[13]:

(1) weaker sufficient convergence conditions in some interesting cases (for example, when $p=1)$;

(2) larger convergence domain;

(3) finer majorizing sequences;

(4) an at least as precise information on the location of the solution.

Note that these advantages are obtained under the same computational cost as in [1]-[5] and [8]-[13] since in practice the computation of the Hölder constant $\ell$ requires the computation of $\ell_{0}$.

In practice, if (2.9) and (3.4) hold, one shall use the finer of the error bounds (see $(3.9)$ and $(5.5))$. If one of $(2.9)$ or (3.4) is violated, then we will use the other one.

Numerical examples further validating the results are also provided in this study.

Acknowledgments. This paper was supported by the Basic Science Research Program through the National Research Foundation of Korea (NRF) funded by the Ministry of Education, Science and Technology (Grant Number: 20120008170).

\section{References}

[1] J. Appell, E. De Pascale, J. V. Lysenko, and P. P. Zabrejko, New results on NewtonKantorovich approximations with applications to nonlinear integral equations, Numer. Funct. Anal. Optim. 18 (1997), no. 1-2, 1-17.

[2] I. K. Argyros, The theory and application of abstract polynomial equations, St. Lucie/CRC/Lewis Publ. Mathematics series, Boca Raton, Florida, 1998.

[3] _ - A unifying local-semilocal convergence analysis and applications for two-point Newton-like methods in Banach space, J. Math. Anal. Appl. 298 (2004), no. 2, 374-397.

[4] _ Concerning the "terra incognita" between convergence regions of two Newton methods, Nonlinear Anal. 62 (2005), no. 1, 179-194.

[5] _ Convergence and Applications of Newton-Type Iterations, Springer, New York, 2008.

[6] I. K. Argyros, Y. J. Cho, and S. Hilout, Numerical Methods for Equations and Its Applications, CRC Press, Taylor and Francis, New York, 2012.

[7] F. Cianciaruso, A further journey in the "terra incognita" of the Newton-Kantorovich method, Nonlinear Funct. Anal. Appl., to appear.

[8] F. Cianciaruso and E. De Pascale, Newton-Kantorovich approximations when the derivative is Hölderian: old and new results, Numer. Funct. Anal. Optim. 24 (2003), no. 7-8, 713-723. 
[9] E. De Pascale and P. P. Zabrejko, Convergence of the Newton-Kantorovich method under Vertgeim conditions: a new improvement, Z. Anal. Anwendvugen 17 (1998), no. $2,271-280$.

[10] L. V. Kantorovich and G. P. Akilov, Functional Analysis, Pergamon Press, Oxford, 1982.

[11] J. V. Lysenko, Conditions for the convergence of the Newton-Kantorovich method for nonlinear equations with Hölder linearizations, Dokl. Akad. Nauk Belarusi 38 (1994), no. 3, 20-24, 122-123.

[12] B. A. Vertgeim, On conditions for the applicability of Newton's method, (Russian) Dokl. Akad. N., SSSR 110 (1956), 719-722.

[13] - On some methods for the approximate solution of nonlinear functional equations in Banach spaces, Uspekhi Mat. Nauk 12 (1957), 166-169 (in Russian); English transl.: Amer. Math. Soc. Transl. 16 (1960), 378-382.

IOANnis Konstantinos Argyros

Department of Mathematics Sciences

CAmeron University

LAWTON, OK 73505, USA

E-mail address: iargyros@cameron.edu

YeOL JE CHO

Department of Mathematics Education and the RINS

Gyeongsang National University

JINJU 660-701, KOREA

E-mail address: yjcho@gnu.ac.kr

SAnthosh George

Department of Mathematical and Computational Sciences

National Institute of Technology Karnataka

INDIA-575025

E-mail address: sgeorge@nitk.ac.in 